\title{
Molecular level investigation of curcumin self-assembly induced by trigonelline and nanoparticle formation
}

\author{
Sweta Sharma ${ }^{1} \cdot$ Ibrahim E. Awad $^{2} \cdot$ Arpita Yadav $^{1} \cdot$ Raymond A. Poirier $^{2} \mathbb{C}$
}

Received: 14 April 2020 / Accepted: 25 July 2020 / Published online: 13 August 2020

(c) King Abdulaziz City for Science and Technology 2020

\begin{abstract}
Nanoparticle-facilitated drug delivery forms the core of medicine nowadays with the drug being delivered right at the target, reducing side effects and enhancing therapeutic value. Nanoparticles derived from natural compounds are further a point of focus being biocompatible and safe by and large. In this study, we have performed HF/6-31G calculations coupled with intermolecular interaction calculations and nanoscale molecular dynamics simulations to investigate self-assemblage in curcumin induced by trigonelline. Similar to recently reported self-assemblage in curcumin induced by sugar, trigonelline, a natural antidiabetic derived from fenugreek, can also induce auto-catalyzed self-assemblage in curcumin to form nanoparticles. It has been shown that these nanoparticles may be utilized for the delivery of drugs with severe side effects especially for diabetic patients with triple benefit of being antidiabetic, biocompatible and safe. As an example, carriage of antidiabetic drug pioglitazone and anticancer drug taxol have been depicted utilizing nanoparticles of curcumin and trigonelline. Twenty five taxol molecules could be comfortably carried in a $50 \mathrm{~nm}$ nanoparticle with an average overall root mean square deviation of $2.89 \AA$ with reference to initial positions. For the first time, this study shows the possibility of developing antidiabetic nanoparticles with plethora of opportunities for diabetic patients. The study is expected to motivate experimental verification and has a long lasting impact in medicinal chemistry.
\end{abstract}

Keywords Curcumin $\cdot$ Trigonelline $\cdot$ Self-aggregation $\cdot$ Drug delivery $\cdot$ Nanoparticle $\cdot$ Taxol

\section{Introduction}

Nanoparticles have revolutionised modern medicine making targeted delivery a reality. Some interesting work in this direction have recently been reported by Karimipour's group, including the effect of nanoparticle diameter on drug delivery (Jafarzadeh et al. 2020). Magnetic nanoparticles are also used for drug delivery and as contrast agent for magnetic resonance imaging (Yadollahpour and Rashidi 2015). Not only in medicine but nanoparticles have also expanded

Electronic supplementary material The online version of this article (https://doi.org/10.1007/s13204-020-01526-4) contains supplementary material, which is available to authorized users.

Raymond A. Poirier

rpoirier@mun.ca

1 Department of Chemistry, University Institute of Engineering and Technology Chhatrapati Shahu Ji Maharaj University, Kanpur 208024, India

2 Department of Chemistry, Memorial University of Newfoundland, St. John's, NL A1B 3X7, Canada the arena of engineering materials. Nanoparticles have been used to modulate thermal conductivity of fluids for use in devices. To attain best usage of nanomaterials in medical devices, biosensors etc., researchers investigate the behaviour of atomic structures in nanoparticles and flow behaviour, heat transfer properties of nanoparticles in atomic channels (Jiang et al. 2020; Abdelmalek et al. 2020; Farzinpour et al. 2020; Liu et al. 2019a, 2020). Accurate nanoscale simulations of the stability of the fluid inside nanochannels have also been reported (Alipour et al. 2019). Thermal conductivity and other physical properties of nanocoolant made from silver nanoparticles produced through green technology and coconut oil have been studied (Sarafraz et al. 2016). Similar experiments have been conducted with $\mathrm{CuO}$ nanosuspension in liquid Indium (Sarafraz and Arjomandi 2018). The convective boiling properties of $\mathrm{CuO}$ nanoparticles in $\mathrm{H}_{2} \mathrm{O}$ / ethylene glycol have been studied at different concentrations of nanoparticles (Sarafraz et al. (2014)). The antibacterial property of $\mathrm{ZnO}$ nanoparticles has been used to develop antibacterial polyethylene which has enormous applications especially in current context of COVID-19 (Galli et al.

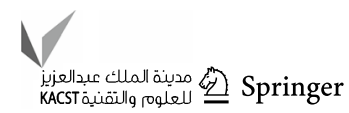


2020). Similarly, Ag and Zn nanoparticles have been utilized to develop bactericidal toothbrushes, the efficiency of which has been measured by Johnson et al. (Johnson et al. 2020) using physical and chemical means.

The above clearly demonstrates the usage and importance of nanoparticles in different walks of life including medicine and modern nanodevices. To curb the toxic aspect related to synthesis and use of metallic nanoparticles efforts are being made worldwide to synthesize them in an eco-friendly environment and preferably from natural, biocompatible and safe material. To cite a few in this direction, we draw attention towards plant-mediated synthesis of Ag nanoparticles (Rajeshkumar and Bharath 2017) and biosynthesis of nanoparticles from fungi and yeast (Moghaddam et al. 2015). This study explores the use of plant product turmeric to form natural, eco-friendly, biocompatible and antidiabetic nanoparticles for drug delivery especially for diabetic patients.

Turmeric is used as a common dietary spice in Asian countries known for its variety of medicinal benefits. It contains $2.5-6 \%$ curcumin, which is a natural polyphenol responsible for its medicinal benefits such as anti-inflammatory, anticancer and can be well tolerated by patients (Teng et al. 2019; Liu et al. 2019). There have been several efforts to utilize the benefits of curcumin, but its low solubility and fast excretion have prohibited its use in medicine (Sharma et al. 2007). However, efforts have been made to enhance its solubility and bio-accessibility (Sharma et al. 2019b; Lin et al. 2019). Recently, curcumin has been the focus of medicinal studies due to its self-assemblage property resulting in the formation of nanoparticles useful in enhancing targeted drug delivery (Wong et al. 2019). Curcumin is a hydrophobic polyphenol which can exist in keto as well as enol form. The enol form is dominant in solution. Self-assemblage is normally observed by surfactant-like amphiphilic molecules. Amphiphilic cyclic or non-cyclic peptides show self-assemblage properties (Ghadiri et al. 1994; Khazanovich et al. 1994; Ghadri et al. 1995; Ghadiri 1995).

This study aims to find out at the molecular level the type of interactions that drive the self-assembly in curcumin. Further, to investigate if the self-assembly can be induced by a natural antidiabetic compound. If feasible, then such nanoparticles or delivery vehicles would be of immense utility for diabetic patients suffering from fatal diseases like cancer. Trigonella foenum-graecum, commonly known as fenugreek is used as a condiment. Fenugreek seeds are also known for their antidiabetic property (Mooventhan and Nivethitha 2017). Trigonelline is one of the main constituents of fenugreek (Ouzir et al. 2016). Similar to recent research findings (Wong et al. 2019), we have first studied self-assemblage in curcumin induced by a sugar mannose. After understanding the governing intermolecular forces in the above case, we have explored the possibility of curcumin self-assemblage in presence of trigonelline as inducing agent. The choice of trigonelline as an inducing agent is based on the fact that it is the main chemical constituent of a natural antidiabetic (Gong et al. 2016). It is a zwitterionic alkaloid, formed by the methylation of the nitrogen atom of niacin (vitamin B3) (Ouzir et al. 2016). This study is expected to help develop biocompatible, safe nanoparticles for efficient delivery of cytotoxic drugs especially for diabetic patients with triple benefits of biocompatibility, safety and antidiabetic nature. In line with this, the delivery of an antidiabetic drug pioglitazone and delivery of an anticancer drug taxol have also been studied.

\section{Methods}

In this work, geometry optimizations at the Hartree-Fock 6-31G level (Ditchfield et al. 1971; Schlegal 1982) along with intermolecular interaction energies, with and without basis set superposition error (BSSE) correction (Boys Bernardi counterpoise correction) (Boys and Bernardi 1970), have been performed to investigate self-assemblage of curcumin molecules in the presence of an inducing agent. Molecular dynamics simulation studies (Haile 2001) have also been performed on self-aggregated forms to understand their stability with time. At first, curcumin, trigonelline and alpha-D-mannose were optimized using Berny's optimization algorithm of the Gaussian software package (Frisch et al. 2016).

\section{Self-aggregation studies using quantum mechanical calculations}

The different complexes of curcumin and inducing agent were geometry optimized from 1:1 to $1: 5$ stoichiometric ratios as shown in Fig. 1. The geometry of the complexes was optimized without any restraint that is, the fragments could reorganize to adopt the most favourable conformations. The 1:5 optimized complex was taken as the repeat unit to slowly build up self-aggregated nanoparticles of larger dimensions as shown in Fig. 2. Single point energy calculations have been carried out on these large nanoparticles. The interaction energies have been computed as follows:

$$
\begin{aligned}
& \Delta E^{(\mathrm{a})}=E_{\text {complex AxBy}}-\left(x \cdot E_{\text {fragment A }}+y \cdot E_{\text {fragment B }}\right), \\
& \Delta E^{(\mathrm{b})}=E_{\text {complex AB }}-\left(\sum^{x} \cdot E_{\text {fragment A }}+\sum^{y} \cdot E_{\text {fragment B }}\right), \\
& \Delta E^{(\mathrm{c})}=E_{\text {complex } \mathrm{AB}}-\left(\sum^{x} \cdot E_{\text {fragment A }}+\sum^{y} \cdot E_{\text {fragment B }}\right),
\end{aligned}
$$




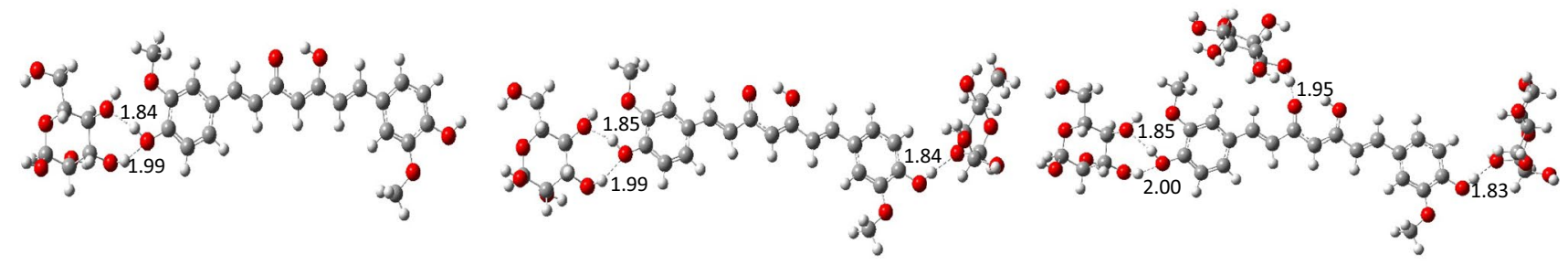

Ratio of Curcumin:mannose $\quad 1: 1$

$1: 2$

1:3

$\Delta \mathrm{E}^{(\mathrm{b})}:-53.9 \mathrm{~kJ} / \mathrm{mol}$
$\Delta \mathrm{E}^{\text {(c) }}:-40.7 \mathrm{~kJ} / \mathrm{mol}$

$\Delta \mathrm{E}^{(\mathrm{b})}:-89.8 \mathrm{~kJ} / \mathrm{mol}$

$\Delta \mathrm{E}^{(\mathrm{b})}:-171.4 \mathrm{~kJ} / \mathrm{mol}$

$\Delta \mathrm{E}^{\text {(c) }}:-\quad-68.7 \mathrm{~kJ} / \mathrm{mol}$

$\Delta E^{(c)}:-134.7 \mathrm{~kJ} / \mathrm{mol}$

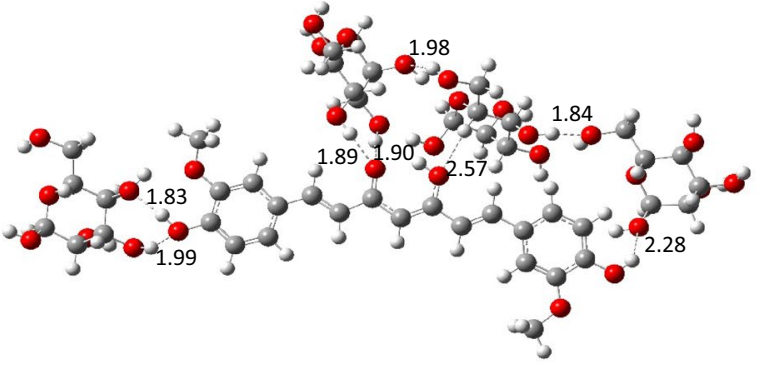

$1: 4$

$\Delta \mathrm{E}^{(\text {b) }}: \quad-282.8 \mathrm{~kJ} / \mathrm{mol}$

$\Delta \mathrm{E}^{\text {(c) }}$ : $\quad-213.7 \mathrm{~kJ} / \mathrm{mol}$

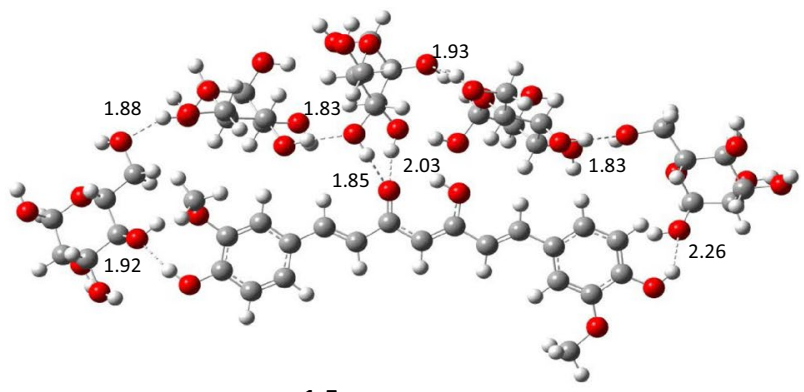

$1: 5$

$\Delta \mathrm{E}^{(\mathrm{b})}: \quad-385.1 \mathrm{~kJ} / \mathrm{mol}$

$\Delta \mathrm{E}^{(\mathrm{c})}:-289.1 \mathrm{~kJ} / \mathrm{mol}$

Fig. 1 Optimized complexes of curcumin and mannose with their respective interaction energies (The H-bonds are shown by dashed line in $\AA$ )

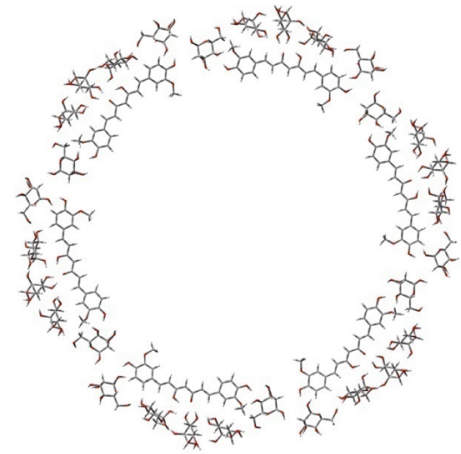

Ratio of curcumin:mannose

$6: 30$

$\Delta \mathrm{E}^{(\mathrm{b})}:-2286.4 \mathrm{~kJ} / \mathrm{mol}$

$\Delta E^{(c)}$ : $-1676.6 \mathrm{~kJ} / \mathrm{mol}$

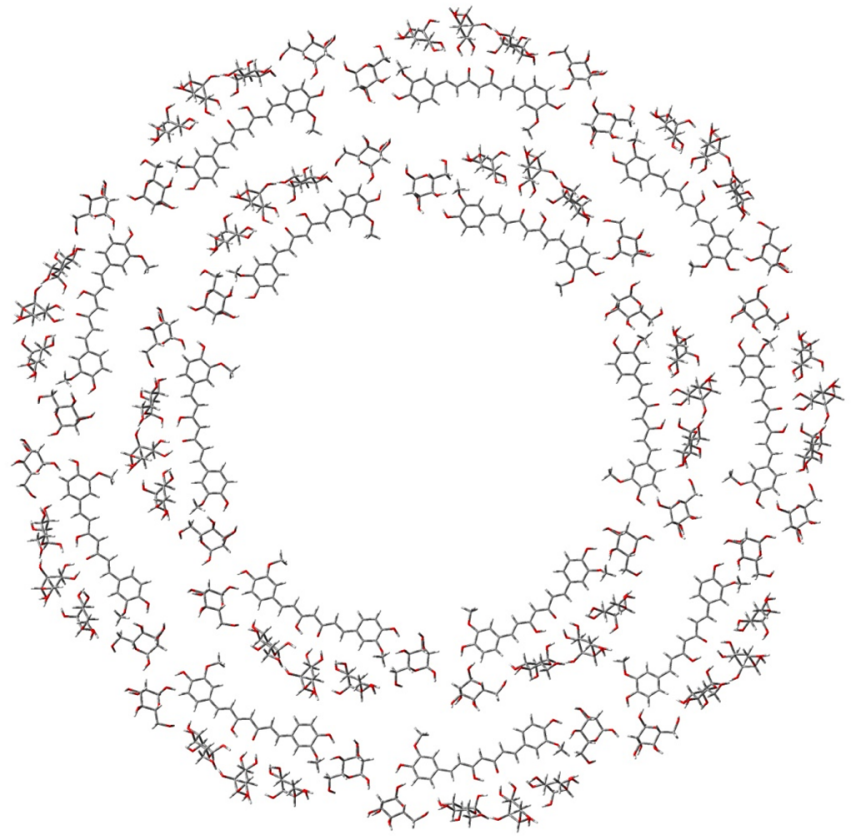

Ratio of curcumin:mannose

15:75

$\Delta \mathrm{E}^{\text {(a) }}: \quad-3584.6 \mathrm{~kJ} / \mathrm{mol}$

Fig. 2 Large curcumin-mannose complexes showing feasibility of curcumin self-assemblage induced by mannose molecules 
where, $\Delta E^{(\mathrm{a})}$ is the interaction energy uncorrected for BSSE and it is with respect to energy of isolated fragments, $\Delta E^{(\mathrm{b})}$ is the interaction energy uncorrected for BSSE and it is with respect to energy of reorganized isolated fragments in the complex and $\Delta E^{(\mathrm{c})}$ is the interaction energy with BSSE correction, that is, counterpoise correction. Equations 1-3 are developed according to ref. (Teng et al. 2019).

\section{Molecular dynamics simulation studies}

To explore the dynamical behaviour and stability over time of self-aggregated nanoparticles, molecular dynamics simulation studies have been performed using the nanoscale molecular dynamics (NAMD) package (Phillips et al. 2005). The CHARMM 36 force field (Klauda et al. 2010; Best et al. 2012) has been used. Force field parameters for curcumin, $\alpha$-D-mannose and trigonelline were generated using CHARMM General force field website at https://www. paramchem.org (Vanommeslaeghe et al. 2010), while force field parameters for pioglitazone and taxol were generated using the SwissParam website at https://www.swissparam.ch (Zoete et al. 2011). The energy minimization was performed for $1 \mathrm{~ns}$. Then the system was subjected to annealing simulation where the temperature was raised from 60 to $300 \mathrm{~K}$ for $2.88 \mathrm{~ns}$. The system was then subjected to equilibration simulation for $10 \mathrm{~ns}$ followed by $100 \mathrm{~ns}$ MD production simulation at $300 \mathrm{~K}$. A NPT ensemble was used. Pressure was maintained at $1 \mathrm{~atm}$ using the Nose-Hoover barostat (Martyna et al. 1994). Long-range interactions were treated using the particle-mesh EWald method (Darden et al. 1993). For all simulations, a $16.0 \AA$ cutoff distance for the Coulomb and van der Waals (vdW) interactions was set and a $15.0 \AA$ cutoff was set for the force-based switching function. The equations of motion were integrated using the r-RESPA multiple time step scheme (Tuckerman et al. 1992) to update short-range interactions for every step and long-range electrostatic interactions for every two steps. The time step of integration was chosen to be 2 fs for all simulations except for the MD production run for which $1 \mathrm{fs}$ was chosen.

The above methodology has been applied to explore selfaggregation of curcumin induced by natural antidiabetic agents with the aim of developing a natural and safe drug delivery system for diabetics to deliver therapeutic agents for fatal diseases like cancer.

\section{Results and discussion}

As mentioned in the methods section, the three molecules, the enol dominant form of curcumin, zwitterionic trigonelline and $\alpha$-D-mannose were first optimized at the HF/6-31G level. Next, different stoichiometric complexes of curcumin and mannose were optimized, results of which are shown in Fig. 1. The oxygens of curcumin, being a polyphenolic compound, act as electrostatically interacting points and also as H-bond donors or acceptors. Five mannose molecules can easily interact in an auto-catalyzed fashion with a curcumin molecule. Although the complexes are geometry optimized, the point of interaction may be governed by initial placement of the molecules. However, the relative change in interaction energies, in other words, the autocatalyzed nature would remain the same. The interaction energies with and without BSSE correction are also collected in Table 1. This led to the understanding that mannose molecules interact attractively with curcumin molecules and therefore larger complexes of curcumin-mannose in 6:30 and 15:75 ratios, where the 1:5 complex was taken as the repeat unit were studied. These complexes are shown in Fig. 2. Single point calculations were performed on these complexes. The calculated interaction energies are given in Table 1. At this point, it is understood that the growth of the complex may not be symmetrical and these structures have been chosen for convenience. However, these results are in agreement with recent experimental observation that sugar can induce selfassemblage of curcumin (Wong et al. 2019). Treating this as the control case it is inferred that curcumin being largely

Table 1 Self-aggregation feasibility of curcumin induced by mannose

\begin{tabular}{|c|c|c|c|}
\hline \\
\hline \multicolumn{4}{|c|}{$\begin{array}{l}\text { Interaction energy* (in } \mathrm{kJ} / \mathrm{mol}) \\
\begin{array}{l}\text { Ratio of } \\
\text { curcumin:mannose }\end{array}\end{array}$} \\
\hline $1: 1$ & -45.2 & -53.9 & -40.7 \\
\hline $1: 2$ & -79.0 & -89.8 & -68.7 \\
\hline $1: 3$ & -139.4 & -171.4 & -134.7 \\
\hline $1: 4$ & -227.7 & -282.8 & -213.7 \\
\hline $1: 5$ & -304.8 & -385.1 & -289.2 \\
\hline $2: 10$ & -604.3 & -766.6 & -569.1 \\
\hline $3: 15$ & -905.5 & -1147.7 & -848.5 \\
\hline $4: 20$ & -1205.8 & -1528.7 & -1127.8 \\
\hline $5: 25$ & -1506.2 & -1910.1 & -1407.6 \\
\hline $6: 30$ & -1802.4 & -2286.4 & -1676.6 \\
\hline $7: 35$ & -2110.5 & -2675.3 & -1966.0 \\
\hline $8: 40$ & -2332.9 & -2978.8 & -2164.6 \\
\hline $9: 45$ & -2470.2 & & \\
\hline $10: 50$ & -2704.9 & & \\
\hline $11: 55$ & -2926.9 & & \\
\hline $12: 60$ & -3063.9 & & \\
\hline $13: 65$ & -3299.0 & & \\
\hline $14: 70$ & -3521.1 & & \\
\hline $15: 75$ & -3584.6 & & \\
\hline
\end{tabular}

$* \Delta E^{(a)}$ is the interaction energy uncorrected for BSSE and it is with respect to energy of isolated fragments, $\Delta E^{(\mathrm{b})}$ is the interaction energy uncorrected for BSSE and it is with respect to energy of reorganized isolated fragments in the complex and $\Delta E^{(\mathrm{c})}$ is the interaction energy with BSSE correction 
hydrophobic, a polar sugar molecule which is of appropriate size can engage curcumin's points of interactions to form successively larger non-covalent complexes or nanoparticles in an auto-catalyzed fashion.

In line with the aim of this research, we have also explored the complexation and self-assemblage of curcumin induced by polar zwitterionic trigonelline which is also of comparable size to mannose and a natural antidiabetic, safe herbal agent. Similar to curcumin-mannose different stoichiometric complexes of curcumin and trigonelline were optimized at the HF/6-31G level results of which are depicted in Fig. 3. Similar to mannose, the trigonelline molecules also interact attractively with curcumin, successively forming larger complexes. A steady increase in interaction energy can be seen with increasing number of trigonelline molecules from 1:1 to 1:5 ratio (from -100.7 to $-556.4 \mathrm{~kJ} /$ $\mathrm{mol}$ ). In addition to H-bonds with the phenolic groups of the curcumin, they also interact with each other electrostatically resulting in more attractive interactions (Table 2). It is, therefore, inferred that trigonelline molecules also interact attractively with curcumin in an auto-catalyzed fashion and may induce its self-aggregation. Larger complexes of curcumin-trigonelline in 6:30 and 15:75 ratios were investigated (Fig. 4). Again the curcumin-trigonelline 1:5 complex was taken as the repeat unit purely for convenience and single point calculation were performed on the large complexes. Interaction energies collected in Table 2 are comparable to curcumin:mannose complexes and indicate good feasibility of trigonelline to act as an inducing agent for the formation of large self-aggregated nanoparticles of curcumin and may, therefore, act as drug delivery vehicles. Again it is emphasized that in reality, aggregation may not be symmetrical.

To further investigate the feasibility of curcumin-trigonelline nanoparticle as a drug delivery vehicle, we have taken the 15:75 ratio complex as the repeat unit (from here on referred as 'one layer'). Layers were now increased one by one to form larger nanoparticles good enough for the carriage of drug molecules. Interaction energy calculations have not been carried out beyond a single layer due to computational limitations. However, the stability of these nanoparticles has been explored through molecular dynamics simulation studies. Figure 5 depicts a nanoparticle formed by stacking of five layers of 15:75 complex of curcumin and trigonelline. The stability of this nanoparticle over time was studied by NAMD simulations the results of which are shown in Fig. 6. A low RMSD in the position of all heavy atoms with respect to initial frame indicates good stability of self-aggregated nanostructure of curcumin over time.

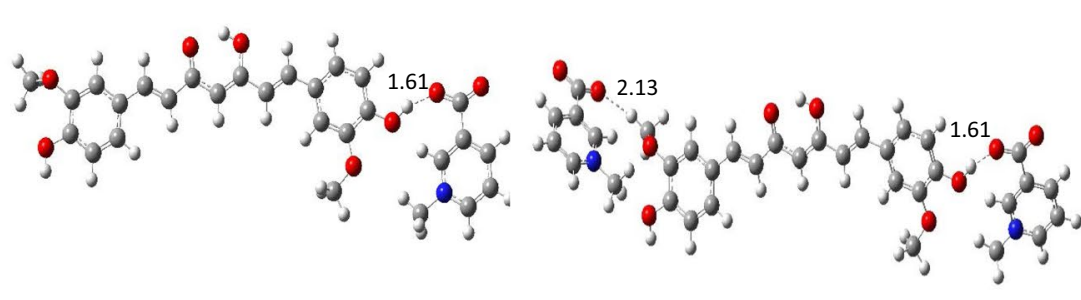

Ratio of curcumin:trigonelline $\quad 1: 1$

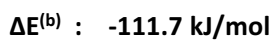

$\Delta \mathrm{E}^{(\mathrm{c})}$ : $-100.7 \mathrm{~kJ} / \mathrm{mol}$
$1: 2$
$\Delta \mathrm{E}^{(\mathrm{b})}:-168.8 \mathrm{~kJ} / \mathrm{mol}$ $\Delta \mathrm{E}^{\text {(c) }}:-148.7 \mathrm{~kJ} / \mathrm{mol}$

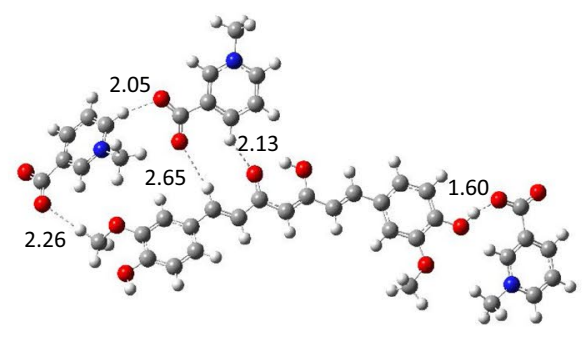

$1: 3$
$\Delta \mathrm{E}^{(\mathrm{b})}:-291.3 \mathrm{~kJ} / \mathrm{mol}$

$\Delta \mathrm{E}^{\text {(c) }}$ : $-254.1 \mathrm{~kJ} / \mathrm{mol}$

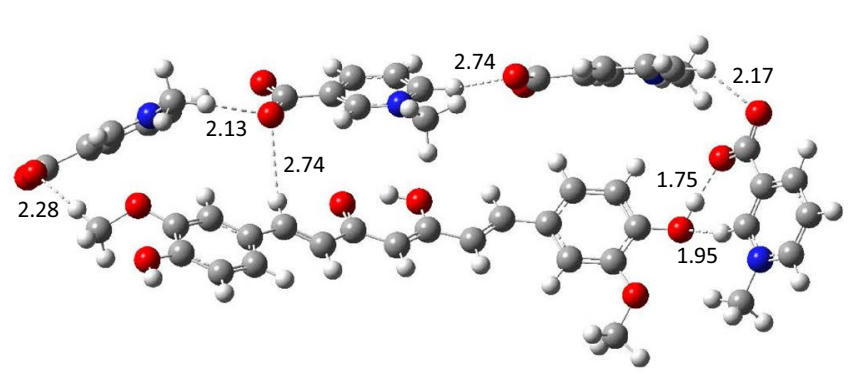

$1: 4$

$\Delta E^{(b)}:-479.2 \mathrm{~kJ} / \mathrm{mol}$

$\Delta E^{(c)}:-416.3 \mathrm{~kJ} / \mathrm{mol}$

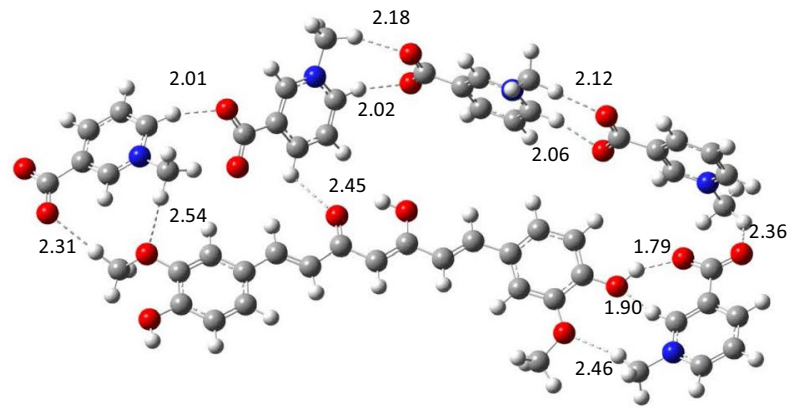

1:5

$\Delta \mathrm{E}^{(\mathrm{b})}: \quad-628.9 \mathrm{~kJ} / \mathrm{mol}$

$\Delta E^{(c)}$ : $\quad-556.4 \mathrm{~kJ} / \mathrm{mol}$

Fig. 3 Optimized complexes of curcumin and trigonellinewith their respective interaction energies (The H-bonds are shown by dashed line in $\AA$ )

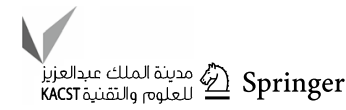


Table 2 Self-aggregation feasibility of curcumin induced by trigonelline

\begin{tabular}{|c|c|c|c|}
\hline \multicolumn{4}{|c|}{ Interaction energy* (in $\mathrm{kJ} / \mathrm{mol}$ ) } \\
\hline $\begin{array}{l}\text { Ratio of } \\
\text { curcumin:trigonelline }\end{array}$ & $\Delta E^{(\mathrm{a})}$ & $\Delta E^{(\mathrm{b})}$ & $\Delta E^{(\mathrm{c})}$ \\
\hline $1: 1$ & -76.4 & -111.7 & -100.7 \\
\hline $1: 2$ & -124.5 & -168.8 & -148.7 \\
\hline $1: 3$ & -244.6 & -291.3 & -254.1 \\
\hline $1: 4$ & -418.0 & -479.2 & -416.3 \\
\hline $1: 5$ & -565.5 & -628.9 & -556.4 \\
\hline $2: 10$ & -1092.4 & -1219.7 & -1066.3 \\
\hline $3: 15$ & -1624.6 & -1816.3 & -1581.9 \\
\hline $4: 20$ & -2158.6 & -2413.8 & -2098.4 \\
\hline $5: 25$ & -2696.4 & -3015.5 & -2619.1 \\
\hline $6: 30$ & -3201.0 & -3583.5 & -3097.8 \\
\hline $7: 35$ & -3722.0 & -4168.6 & -3608.1 \\
\hline $8: 40$ & -4320.9 & -4831.6 & -4193.1 \\
\hline $9: 45$ & -4949.3 & & \\
\hline $10: 50$ & -5504.2 & & \\
\hline $11: 55$ & -6109.1 & & \\
\hline $12: 60$ & -6739.0 & & \\
\hline $13: 65$ & -7296.2 & & \\
\hline $14: 70$ & -7905.7 & & \\
\hline $15: 75$ & -8571.9 & & \\
\hline
\end{tabular}

$* \Delta E^{(\mathrm{a})}$ is the interaction energy uncorrected for BSSE and it is with respect to energy of isolated fragments, $\Delta E^{(\mathrm{b})}$ is the interaction energy uncorrected for BSSE and it is with respect to energy of reorganized isolated fragments in the complex and $\Delta E^{(\mathrm{c})}$ is the interaction energy with BSSE correction

The segregated RMSDs of all curcumin molecules and all trigonelline molecules, which are also low, are shown on the same plot indicating usual movement of atoms at $300 \mathrm{~K}$ and no instability. To further validate the integrity and stability of the nanoparticles over time, snapshots of the system are shown at 20 ns intervals.

After understanding the stability of the nanoparticle, we have explored the utility of this nanoparticle in drug delivery. Figure 7 depicts the results for the carriage of antidiabetic drug pioglitazone. The first and last frame of NAMD simulation indicates good carriage and no premature expulsion of the drug. The low RMSD in the position of all heavy atoms indicates usual movement at $300 \mathrm{~K}$. Segregated RMSDs also indicate good stability of curcumin and trigonelline molecules. The pioglitazone molecules are small and can freely move around in the pore of the nanoparticle formed by self-aggregation of curcumin and trigonelline molecules. This movement is indicated by fluctuations in positions of pioglitazone atoms keeping the overall RMSD low (Fig. 7).

We have also investigated carriage of an anticancer drug taxol results for which are shown in Fig. 8. Taxol seems to be quite stable inside the nanoparticle with very low fluctuations in its position. Overall RMSD in position of all heavy atoms indicates usual movement at room temperature and no premature expulsion of the drug. Taxol being a larger molecule, its movement inside the pores of the nanoparticle is very restricted as evident from the RMSD of about $1 \AA$. These results encouraged us to pursue a self-aggregated nanoparticle of about $50 \mathrm{~nm}$ dimension carrying 25 taxol molecules. Simulation results for this system are shown in Fig. 9 and also available as video in Supplement 1. Figure 9 further validates smooth carriage of 25 taxol molecules without any premature expulsion. An average (over $100 \mathrm{~ns}$ ) overall RMSD of $2.89 \AA$ was observed. Segregated average RMSD's are $3.17 \AA$ for curcumin molecules, $2.63 \AA$ for taxol molecules and $2.74 \AA$ for trigonelline molecules.

The study thus highlights the possibility of utilizing selfaggregated form of curcumin and trigonelline for delivery of drugs especially for diabetic patients.

\section{Conclusions}

In this work, we have shown self-assemblage of curcumin induced by zwitterionic trigonelline molecules forming nanoparticles which can possibly be utilized for carriage and delivery of toxic drugs with severe side effects. Nanoscale molecular dynamics simulation studies have been utilized to show carriage of antidiabetic drug pioglitazone and anticancer drug taxol. $100 \mathrm{~ns}$ simulation of a nanoparticle with a $50 \mathrm{~nm}$ diameter carrying 25 taxol molecules shows stability with a low average RMSD value of $2.89 \AA$ for the entire system. There was no tendency of breakage of the nanoparticle or expulsion of the drug. Since trigonelline itself possesses antidiabetic properties such nanoparticles would be particularly beneficial for diabetic patients. Trigonelline being polar is expected to overcome solubility issues with curcumin. This study reports an antidiabetic nanoparticle for the first time and is expected to lead to long term medicinal benefits on experimental verification. 


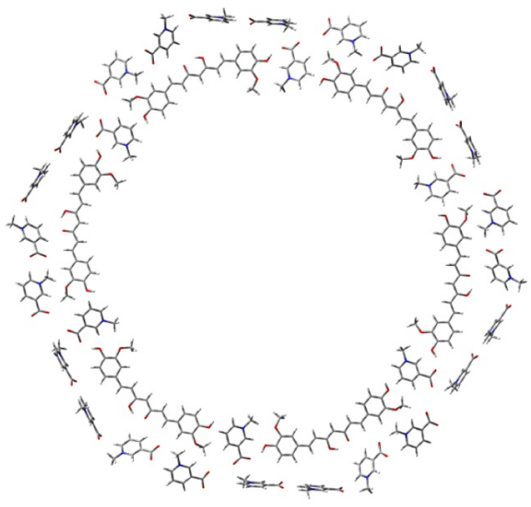

Ratio of curcumin:trigonelline $\quad 6: 30$

$\Delta E^{(b)}:-3583.5 \mathrm{~kJ} / \mathrm{mol}$

$\Delta \mathrm{E}^{(\mathrm{c})}:-3097.8 \mathrm{~kJ} / \mathrm{mol}$

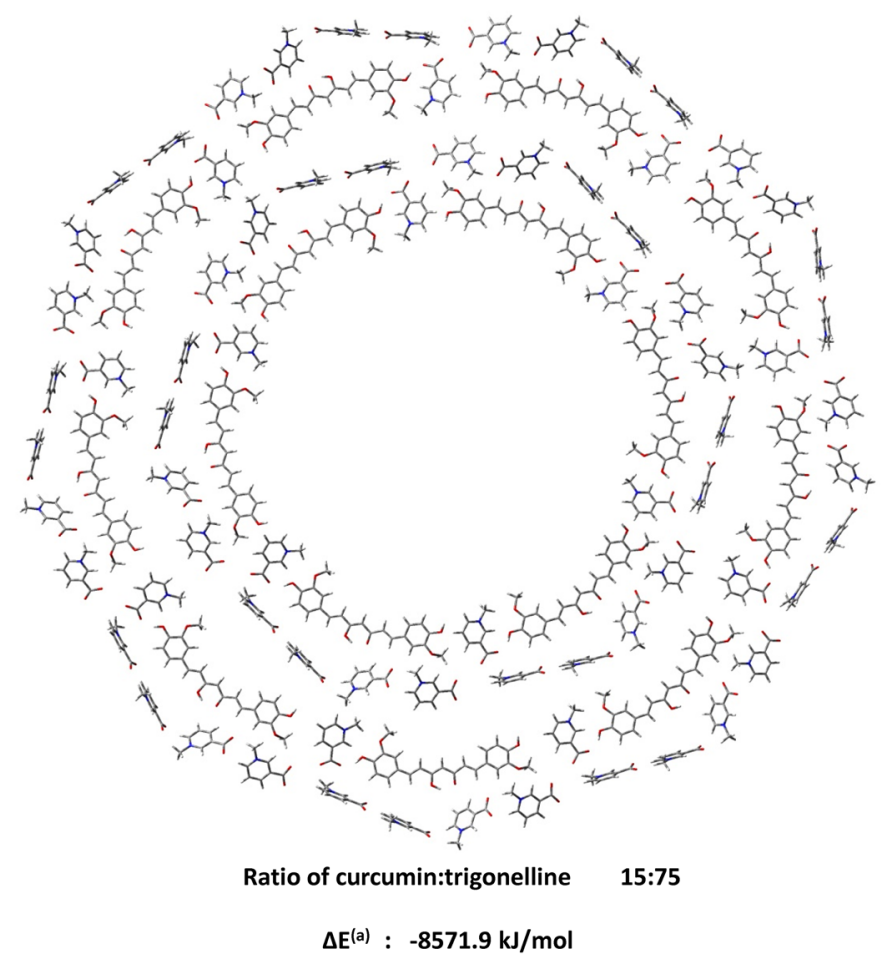

Fig. 4 Large curcumin-trigonelline complexes showing feasibility of curcumin self-assemblage induced by trigonelline molecules

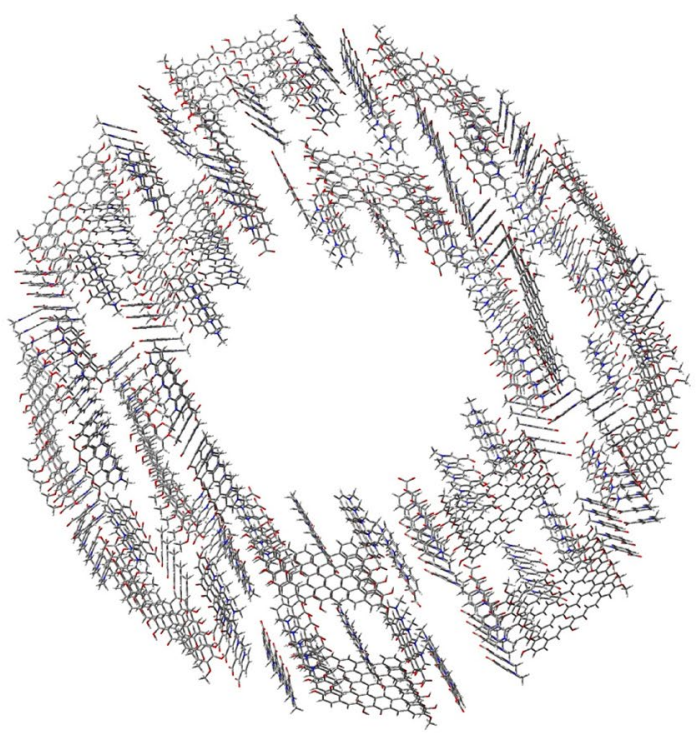

Top view
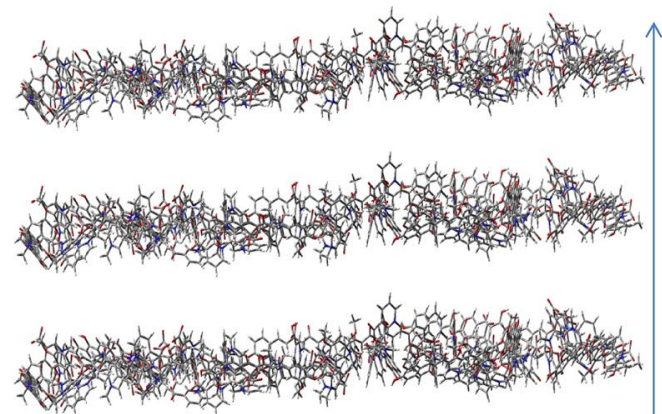

$\sim 87.81 \AA$

Fig. 5 Stacking of five layers (each layer is comprised of a complex of curcumin:trigonelline ratio of 15:75) rendering a small approximately $9 \mathrm{~nm} \times 9.5 \mathrm{~nm}$ nanoparticle for the carriage of drug 


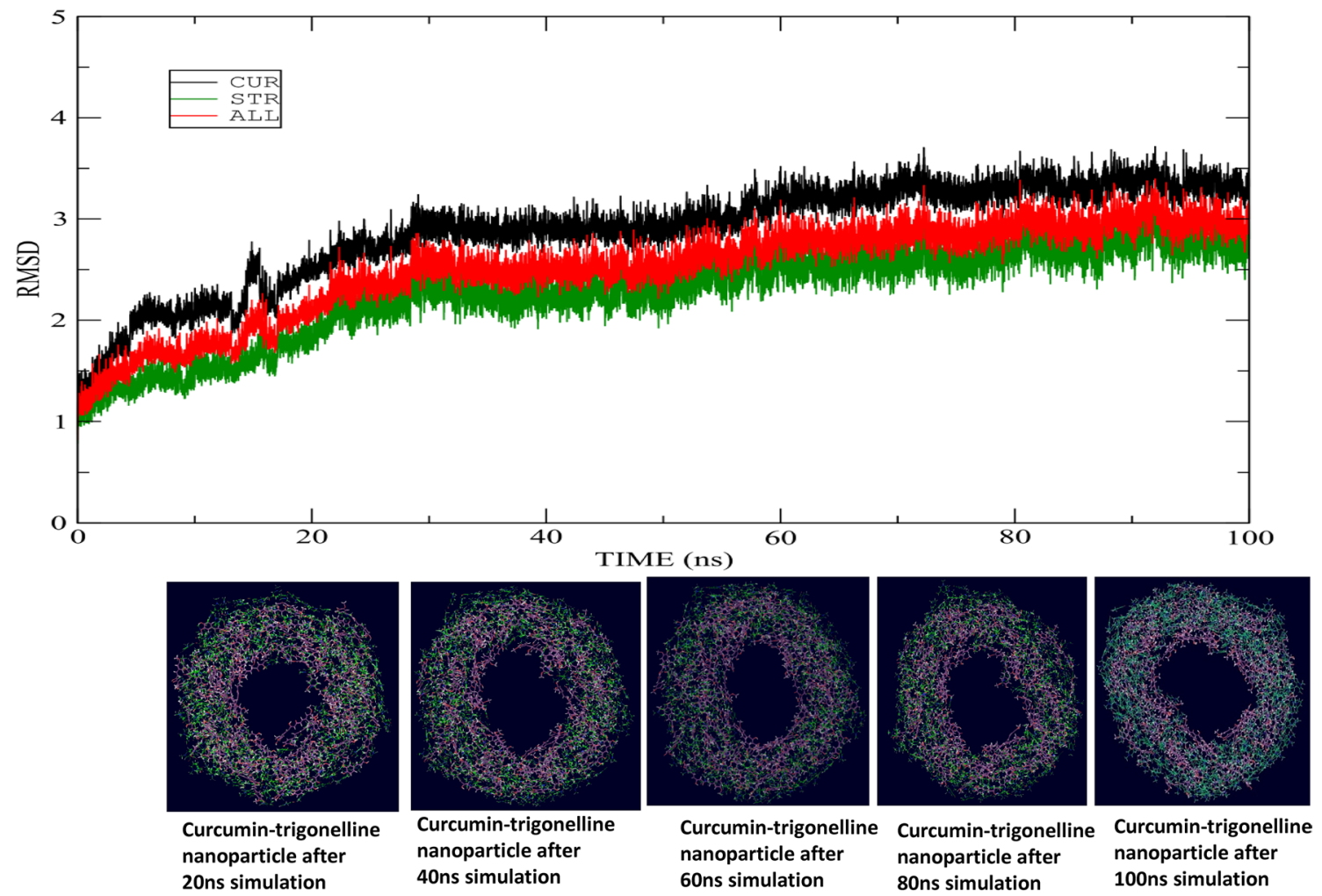

Fig. 6 Root mean square deviation (in $\AA$ ) in position of all heavy atoms (shown in red) with respect to initial frame, for curcumin molecules (shown in black) and for trigonelline molecules (shown in green) in the nanoparticle 

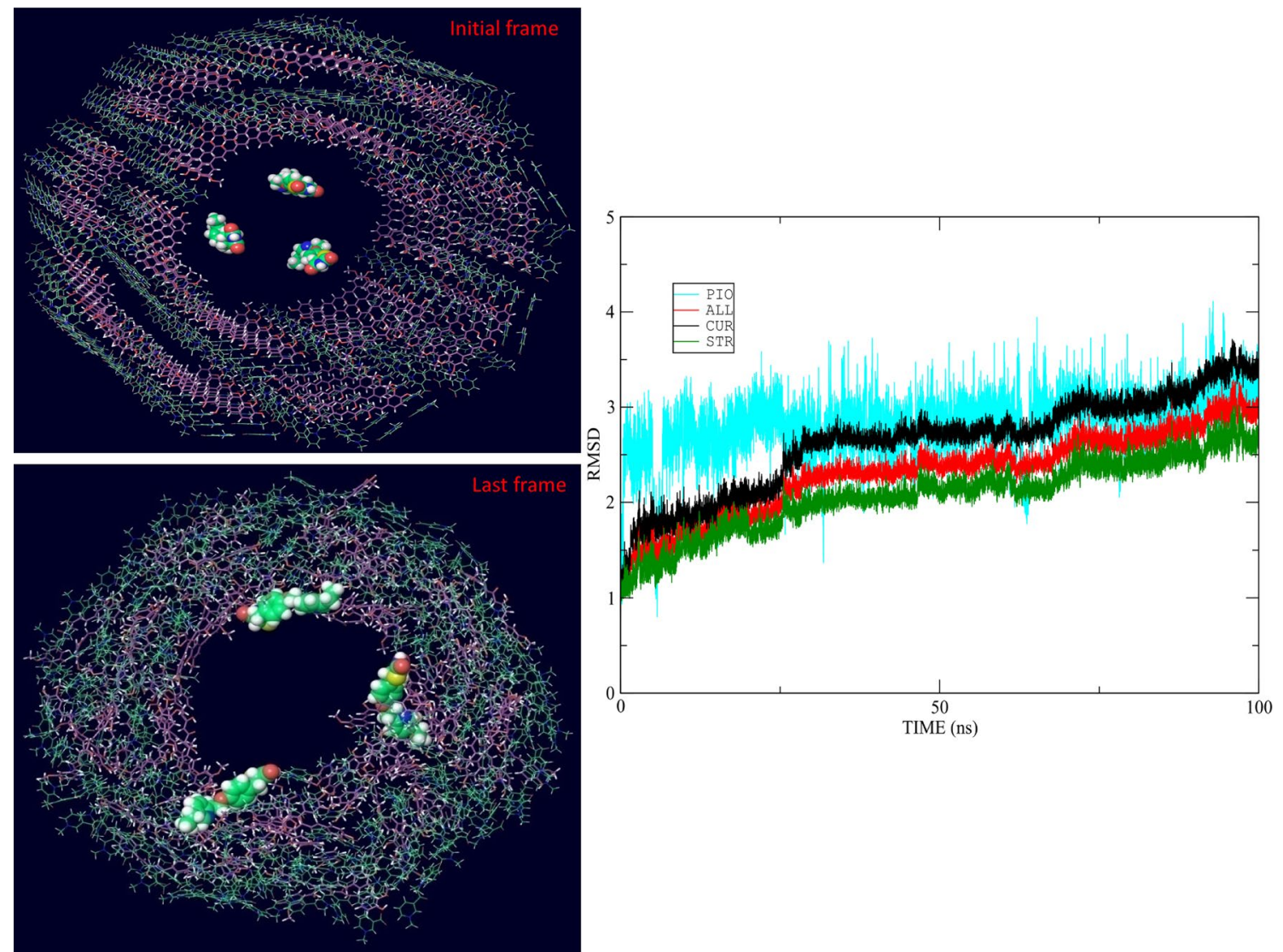

Curcumin-trigonelline nanoparticle carrying pioglitazone drug

Fig. 7 Root mean square deviation (in $\AA$ ) in position of all heavy atoms (shown in red) with respect to initial frame, for curcumin molecules (shown in black), for trigonelline molecules (shown in green) and for pioglitazone molecules (shown in cyan) in the nanoparticle 

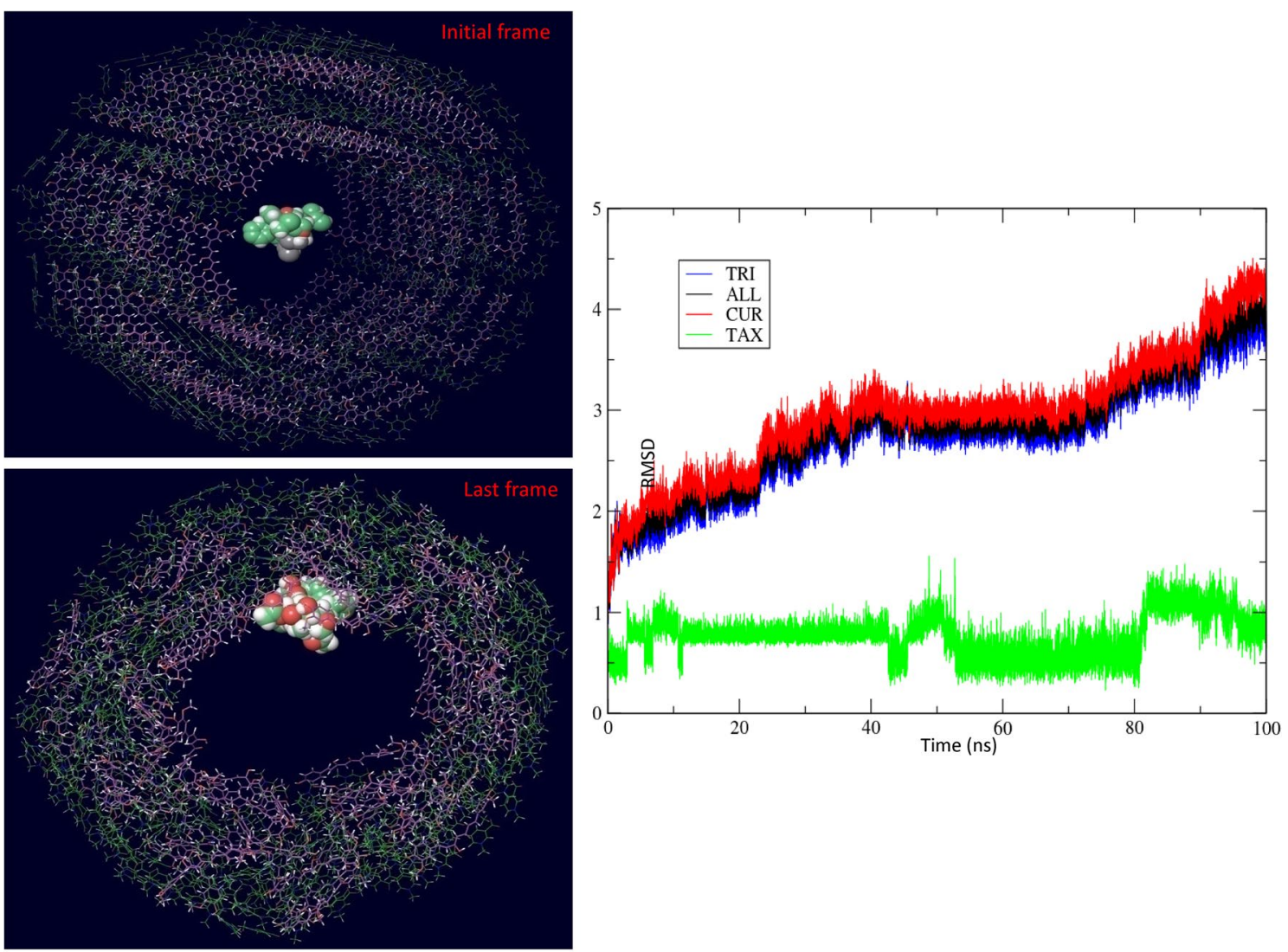

Curcumin-trigonelline nanoparticle carrying taxol drug

Fig. 8 Root mean square deviation (in $\AA$ ) in position of all heavy atoms (shown in black) with respect to initial frame, for curcumin molecules (shown in red), for trigonellinemolecules (shown in blue) and for taxol molecule (shown in green) in the nanoparticle 

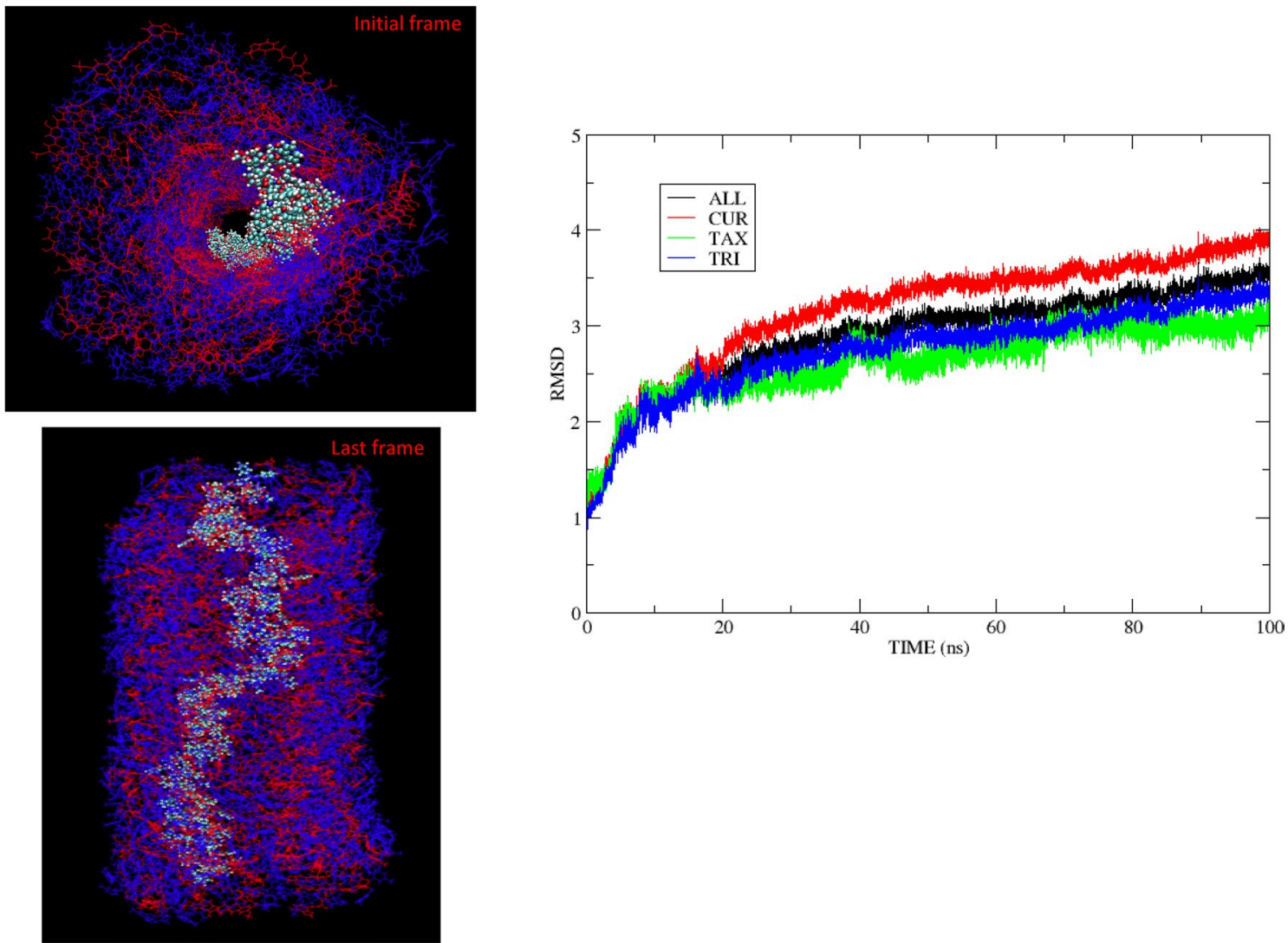

Fig. 9 Root mean square deviation (in $\AA$ ) in position of all heavy atoms (shown in black) with respect to initial frame, for curcumin molecules (shown in red), for trigonelline molecules (shown in blue) and for taxol molecules (shown in green) in the nanoparticle

Acknowledgements We gratefully acknowledge Compute Canada for computer time. R.A.P. is grateful to the Natural Sciences and Engineering Research Council of Canada (NSERC) for financial support. A.Y. and S.S. express their thanks to Science and Engineering Research Board, New Delhi for research grant number EMR/2016/000769. S.S. gratefully acknowledges Senior Research Fellowship from SERB. A.Y. and S.S. thank University Institute of Engineering and Technology, CSJM University, Kanpur for providing infrastructural support.

\section{Compliance with ethical standards}

Conflict of interest On behalf of all authors, the corresponding author states that there is no conflict of interest.

\section{References}

Abdelmalek Z, D'Orazio A, Karimipour A (2020) The effect of nanoparticle shape and microchannel geometry on fluid flow and heat transfer in a porous microchannel. Symmetry 12:591-619

Alipour P, Toghraie D, Karimipour A (2019) Investigation the atomic arrangement and stability of the fluid inside a rough nanochannel in both presence and absence of different roughness by using of accurate nanoscale simulation. Phys A 524:639-660

Best RB, Zhu X, Shim J, Lopes PEM, Mittal J, Feig M, MacKerell AD (2012) Optimization of the additive CHARMM All-atom protein force field targeting improved sampling of the backbone $\varphi, \psi$ and side-chain $\chi^{1}$ and $\chi^{2}$ dihedral angles. J Chem Theory Comput 8(9):3257-3273

Boys SF, Bernardi F (1970) Calculation of small molecular interactions by differences of separate total energies some procedures with reduced errors. Mol Phys 19:553-566

Darden T, York D, Pederson L (1993) Particle mesh EWalld: an $\mathrm{N} \log (\mathrm{N})$ method for EWald sums in large systems. J Chem Phys 98:10089-10092

Ditchfield R, Hehre WJ, Pople JA (1971) Self-consistent molecularorbital methods. IX. An extended gaussian-type basis for molecular-orbital studies of organic molecules. J Chem Phys 54:724

Farzinpour M, Toghraie D, Mehmandoust B, Aghadavoudi F, Kasrimipour A (2020) Molecular dynamics simulation of ferronanofluid behaviour in a nanochannel in the presence of constant and timedependent magnetic fields. J Therm Anal Calorim. https://doi. org/10.1007/S10973-020-09846-x

Frisch MJ, Trucks GW, Schlegel HB, Scuseria GE, Robb MA, Cheeseman JR, Scalmani G, Barone V, Petersson GA, Nakatsuji H, Li X, Caricato M, Marenich AV, Bloino J, Janesko BG, Gomperts R, Mennucci B, Hratchian HP, Ortiz JV, Izmaylov AF, Sonnenberg JL, Williams-Young D, Ding F, Lipparini F, Egidi F, Goings J, Peng B, Petrone A, Henderson T, Ranasinghe D, Zakrzewski VG, Gao J, Rega N, Zheng G, Liang W, Hada M, Ehara M, Toyota K, Fukuda R, Hasegawa J, Ishida M, Nakajima T, Honda Y, Kitao O, Nakai H, Vreven T, Throssell K, Montgomery JA Jr, Peralta JE, Ogliaro F, Bearpark MJ, Heyd JJ, Brothers EN, Kudin KN, Staroverov VN, Keith TA, Kobayashi R, Normand J, Raghavachari 
K, Rendell AP, Burant JC, Iyengar SS, Tomasi J, Cossi M, Millam JM, Klene M, Adamo C, Cammi R, Ochterski JW, Martin RL, Morokuma K, Farkas O, Foresman JB, Fox DJ (2016) Gaussian 16, Revision C.01. Gaussian, Inc., Wallingford

Galli R, Hall MC, Breitenbach ER, Colpani GL, Zanetti M, Muneron de Mello JM, Silva LL, Fiori MA (2020) Antibacterial polyethylene-ethylene vinyl acetate polymeric blend by incorporation of zinc oxide nanoparticles. Polym Testing 89:106554-106564

Ghadiri MR (1995) Self-assembled nanoscale tubular ensembles. Adv Mater 7:675-677

Ghadiri MR, Granja JR, Buehler LK (1994) Artificial transmembrane ion channels from self-assembling peptide nanotubes. Nature (London) 369:301-304

Ghadri MR, Kobayashi K, Granja JR, Chadha RK, McRee DE (1995) The Structural and thermodynamic basis for the formation of self-assembled peptide nanotubes. Angew Chem Int Ed (Engl) 34:93-95

Gong J, Fang K, Dong H, Wang D, Hu M, Lu E (2016) Effect of fenugreek on hyperglycaemia and hyperlipidemia in diabetes and prediabetes: a meta-analysis. J Ethnopharmacol 194:260-268

Haile JM (2001) Molecular dynamics simulation: elementary methods. Wiley, Hoboken (ISBN0-471-18439-x)

Jafarzadeh S, Nasiri Sadr A, Kaffash E, Gondarzi S, Golab E, Karimipour A (2020) The effect of hematocrit and nanoparticle's diameter on hemodynamic parameters and drug delivery in abdominal aortic aneurysm with consideration of blood pulsatile flow. Comp Methods Prog Biomed 195:105545-105557

Jiang Y, Dehghan S, Karimipour A, Toghraie D, Li Z, Tlili I (2020) Effect of copper nanoparticles on thermal behaviour of water flow in a zig-zag nanochannel using molecular dynamics simulation. Int Commun Heat Mass Trans 116:104652-104660

Johnson CR, Tran MN, Michelitsch LM, Abraham S, Hu J, Gray KA, Hartman EM (2020) Nano-enabled, antimicrobial toothbrushesHow physical and chemical properties relate to antibacterial capabilities. J Hazard Mater 396:122445-122457

Khazanovich N, Granja JR, McRee DE, Milligan RA, Ghadiri MR (1994) Nanoscale tubular ensembles with specified internal diameters design of a self-assembled nanotube with a 13-.ANG pore. J Am Chem Soc 116:6011

Klauda B, Venable RM, Freites JA, O'Connor JW, Tobias DJ, Mondragon-Ramirez C, Vorobyov I, MacKerell AD, Pastor RW (2010) Update of the CHARMM All-atom additive force field for lipids: validation on six lipid types. J Phys Chem B 114(23):7830-7843

Lin JT, Ye QB, Yang QJ, Wang GH (2019) Hierarchial bioresponsive nanocarriers for codelivery of curcumin and doxorubicin, colloids and surfaces B. Biointerfaces 180:93-101

Liu WI, Malekahmadi O, Bagherzadeh SA, Ghashang M, Karimipour A, Hasani S, Tlili I, Goodarzi M (2019a) A novel comprehensive experimental study concerned graphene oxide nanoparticles dispersed in water: synthesise, characterisation, thermal conductivity measurement and present a new approach of RLSF neural network. Int Commun Heat Mass Transf 109:104333-104349

Liu Y, Sun H, Makabael B, Cui Q, Li J, Su C, Ashby IRCR (2019b) The targeting of non-coding RNAs by curcumin: facts and hopes for cancer therapy. Oncol Rep 42:20-34

Liu X, Toghraie D, Hekmatifar M, Akbari OA, Karimipour A, Afrand M (2020) Numerical investigation of nanofluid laminar forced convection heat transfer between two horizontal concentric cylinders in the presence of porous medium. J Therm Anal Calorim. https://doi.org/10.1007/S10973-020-09406-3

Martyna GJ, Tobias DJ, Klein ML (1994) Constant pressure molecular dynamics algorithms. J Chem Phys 101:4177-4189
Moghaddam AB, Namvar F, Moniri M, Tahir PM, Azizi S, Mohamad R (2015) Nanoparticles biosynthesized by fungi and yeast: a review of their preparation, properties and medical applications. Molecules 20:16540-16565

Mooventhan A, Nivethitha L (2017) A narrative review on evidencebased antidiabetic effect of fenugreek (Trigonella foenum-graecum). Int J Nutr Pharmacol Neurol Dis 7:84-87

Ouzir M, Bairi KE, Amzazi S (2016) Toxicology properties of fenugreek (Trigonella foenum-graecum). Food Chem Toxicol 96:145-154

Phillips JC, Braun R, Wang W, Gumbart J, Tajkhorshid E, Villa E, Chipot C, Skeel RD, Kale L, Schulten K (2005) Scalable molecular dynamics with NAMD. J Comput Chem 26:1781-1802

Rajeshkumar S, Bharath LV (2017) Mechanism of plant-mediated synthesis of silver nanoparticles-a review on biomolecules involved, characterisation and antibacterial activity. Chemico-Biol Interac 273:219-227

Sarafraz MM, Arjomandi M (2018) Thermal performance analysis of a microchannel heat sink cooling with copper oxide-indium $(\mathrm{CuO} / \mathrm{In})$ nano-suspensions at high-temperatures. Appl Therm Eng 137:700-709

Sarafraz MM, Hormozi F, Kamalgharibi M (2014) Sedimentation and convective boiling heat transfer of $\mathrm{CuO}$-water/ethylene glycol nanofluids. Heat Mass Transf 50:1237-1249

Sarafraz MM, Arya A, Nikkhah V, Hormozi F (2016) Thermal performance and viscosity of biologically produced silver/coconut oil nanofluids. Chem Biochem Eng Q 30(4):489-500

Schlegal HB (1982) Optimization of equilibrium geometries and transition structures. J Comp Chem 3:214-218

Sharma RA, Steward WP, Gescher AJ (2007) Pharmacokinetics and pharmacodynamics of curcumin. Adv Exp Med Biol 595:453-470

Sharma M, Sharma S, Wadhwa J (2019) Improved uptake and therapeutic intervention of Curcumin via designing binary lipid nanoparticulate formulation for oral delivery in inflammatory bowel disorder. Artif Cells Nanomed Biotechnol 47(1):45-55

Teng CF, Yu CH, Chang HY, Hsieh WC, Wu TH, Lin JH, Wu HC, Jeng LB, Su IJ (2019) Chemopreventive effect of phytosomal curcumin on Hepatitis B virus-related hepatocellular carcinoma in a transgenic mouse model. Sci Rep 9:103378

Tuckerman M, Berne BJ, Martyna GJ (1992) Reversible multiple time scale molecular dynamics. J Chem Phys 97(3):1990-2001

Vanommeslaeghe K, Hatcher E, Acharya C, Kundu S, Zhong S, Shim J, Darian E, Guvench O, Lopes P, Vorobyov I, MacKerell AD (2010) CHARMM general force field: a force field for drug-like molecules compatible with the CHARMM all-atom additive biological force fields. J Comput Chem 31(4):671-690

Wong S, Zhao J, Cao C, Wong CK, Kuchel RP, Luca SD, Hook JM, Garvey CJ, Smith S, Ho J, Stenzel MH (2019) Just add sugar for carbohydrate induced self-assembly of Curcumin. Nat commun 10:582

Yadollahpour A, Rashidi S (2015) Magnetic nanoparticles: a review of chemical and physical characteristics important in medical applications. Orient J Chem 31(Spl. Edn.):25-30

Zoete V, Cuendet MA, Grosdidier A, Michielin O (2011) SwissParam: a fast force field generation tool for small organic molecules. $\mathrm{J}$ Comput Chem 32(11):2359-2368

Publisher's Note Springer Nature remains neutral with regard to jurisdictional claims in published maps and institutional affiliations. 